\title{
Mechanotransduction in blood cells
}

\author{
Fumihiko Nakamura* \\ Health Sciences Platform, Tianjin University, Tianjin 300072, China
}

\begin{abstract}
Blood cells are subjected to various mechanical forces including pressure, flow, shear force, gravity, and forces acting against them with varying stiffness (eg. blood vessel wall). Scientists have discovered that these forces have profound effects on cellular growth, differentiation, secretion of cytokines, cell death, and migration. These processes are called mechanotransduction, a conversion of mechanical forces to biochemical signals. In this article the author reviews biophysical forces that affect biological functions of blood cells and their responses in normal physiology and pathophysiology. Although input (forces) and output (cellular responses) have been well studied by utilizing recently developed various force-generating devices, the molecular mechanism of mechanotransudction is still a mystery. This is because reconstructing molecular interaction in the presence of mechanical forces in vitro is highly challenging and until now the molecular dynamics involved in structural changes caused by these forces are largely unknown. Nevertheless, the author has reviewed a few examples of potential structural effects on the molecular mechanism of mechanotransduction.
\end{abstract}

Keywords: mechanotransduction, blood, mechanical force, pressure, gravity, shear flow, stiffness

\section{INTRODUCTION}

Animal cells are far from simple static building blocks. They are both internally and externally dynamic, moving and changing their shapes when they divide, differentiate, and migrate to build or repair a living organism. These processes inevitably rely on active changes of a cell's mechanics ${ }^{[1,2]}$. For example, white blood cells need to generate an internal force at specific locations to crawl through tissues while chasing down and ingesting pathogens. In red blood cells (RBCS), "storage lesions" changes the deformability , which leads to pathological changes in hemodynamics, thereby limiting transfusion ability ${ }^{[3]}$. Because the mechanical properties of cells and tissues can also be

*Correspondence to: Fumihiko Nakamura Health Sciences Platform, Tianjin University, Tianjin 300072, China

E-mail: fnakamura@tju.edu.cn

The authors declared that they do not have anything to disclose regarding funding or conflict of interest with respect to this manuscript. affected by disease and inflammation ${ }^{[4-6]}$, knowledge of cell mechanics is essential for elucidating many fundamental aspects of cell behavior in normal and pathologic conditions, and can be applied for diagnostic purposes.

Cells also respond to external mechanical forces in their environment. Vascular cells undergo morphological changes in response to alterations in the shear stresses that are imposed on them by blood flow, and these responses have profound implications in the physiological function of blood vessels, and in disorders such as in atherosclerosis and thrombosis ${ }^{[7,8]}$. In some experimental studies (dependent on the stiffness of substrates) stem cells differentiate into different cells, namely neurons on soft matrices that mimic brain tissue, myoblasts on stiffer matrices that mimic muscle, and osteoblasts on rigid matrices that mimic bone ${ }^{[9]}$. Therefore, a detailed understanding of how mechanical forces elicit biochemical signals (mechanotransduction) has important implications in human health and disease.

A PubMed search of "mechanotransduction" hits 
over 7,000 reports and further filtering with "blood cells" narrows the results down to about 1,000. The accumulation of literature has exponentially increased in the past ten years because not only more scientists have been recognizing the importance of physical forces on biological function, but also due to the development of experimental devices and materials such as stretching devices and stiffness-controlled matrix. Using these devices, scientists discovered that mechanical forces directly or indirectly affect cellular functions. In blood cells, mechanotransduction is implicated in hypotension, hypertension and haemorrhaging ${ }^{[10]}$ and maintaining normal physiology ${ }^{[11]}$.

Endothelial cells on the luminal surface of blood vessels are activated by force and have effects on blood cells: for example, disturb flow and hypercholesterolemia synergistically promote T-lymphocyte activation $^{[12]}$. Endothelial cleft shear stress inhibits neutrophil transmigration via a NO-dependent mech-
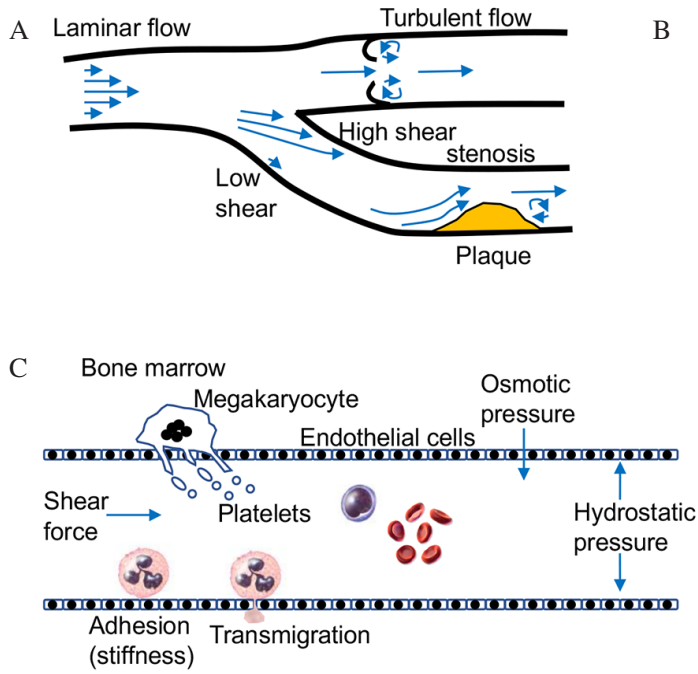

B

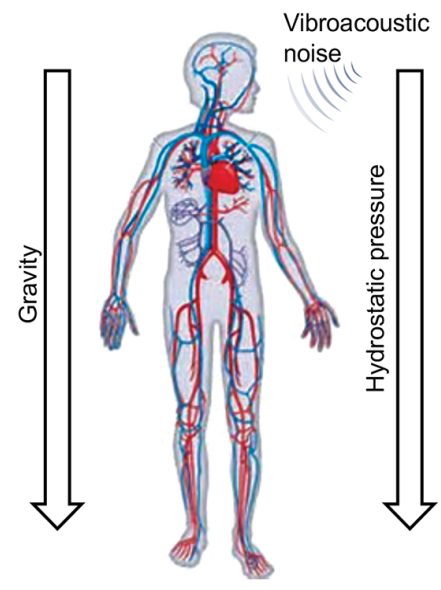

Fig. 1. Schematic diagram of hemodynamic forces acting on blood cells. (A) Flow and shear patterns in different parts of the vascular tree. In regions, where vessels branch, shear stress varies depending on vessel diameter and angle. Plaque also disturbs laminar flow. (B) Blood pressure depends on volume of blood forced into the vessel, distensibility of vessel walls. Therefore, the pressure is pulsatile and decreases over $90 \%$ from aorta to vena cava. In a standing position, hydrostatic pressure caused by gravity increases blood pressure below the heart and decreases pressure above it. (C) Blood cells experience shear stress and pressure in blood stream. Leukocyte, for example, adheres on endothelium lines on the inner wall of vessels, senses substrate stiffness, and generates traction force to migrate. When it transmigrates, mechanical properties of the cells dramatically change.

Blood pressure is generated by the heart pumping blood into the arteries as well as by the contraction and resistance of the arteries. Blood pressure decreases more than $90 \%$ from aorta (normally $\sim 120 \mathrm{mmHg} / 16$ $\mathrm{kPa})$ to the vena cava ${ }^{[19]}$. Hydrostatic pressure generated by gravity increases blood pressure below the heart and decreases pressure above it in a human in standing position (Fig. 1). Osmotic pressure also influences blood pressure depending on the tissues that surround the vessels. Therefore, blood cells are able to act normally in a wide range of blood pressures in the absence of disease and require such changes to maintain normal in vivo conditions. Nevertheless cells can respond to hypo- or hyper-blood pressures when sustained for an unusual period of time with abnormal force configuration. Anomalous alternation of blood pressure (eg. diving) elicits dramatic cardiorespiratory and endocrine responses ${ }^{[20]}$.

Shear stress generated by blood flow has been proved to influence cellular functions and phenotypes, and has been extensively tested in the laboratory using shear flow devices ${ }^{[21,22]}$. Studies indicate that flow and shear pattern change depend on the vascular tree shape. High shear generally protects from athero- 
sclerosis, whereas low shear and turbulent flow may lead to wall thickening and atherosclerotic plaque formation which disturbs laminar flow ${ }^{[23]}$ (Fig. 1). Balloon angioplasty and stent implantation also perturb the flow ${ }^{[24]}$. Even in normal physiology, shear stress in blood vessels varies dramatically from nearly zero to $53 \mathrm{dyn} / \mathrm{cm}^{2}(\sim 1,600 / \mathrm{s}$ share rate) due to changes in blood flow rate ${ }^{[25]}$. However, aberrantly low, or high shear stress for abnormal periods of time lead to diseases such as atherosclerosis ${ }^{[26]}$. Using high shear, present at a lesion area, it is possible to specifically deliver a drug to the site using shear-activated nanoparticles ${ }^{[27]}$.

The gravitational force is minor at the cellular length scale when compared to typical intramolecular forces. Yet, spaceflight has been shown to induce profound effects on lymphocyte activation ${ }^{[28,29]}$ and other cellular responses ${ }^{[30]}$. This field of research will be more and more important as humans increasingly explore and live in a space ${ }^{[31]}$.

Infrasound $(0-20 \mathrm{~Hz})$ and low-frequency noise (20-500 Hz) causes vibroacoustic disease (VAD) and increases in CD8+ and CD4+ T lymphocytes ${ }^{[32,33]}$. However, although VAD is clearly a mechanotransduction disease, how the sound-wave force leads to $\mathrm{VAD}$ remains enigmatic.

When the erythrocyte squeezes into a tiny capillary, its body becomes stretched markedly. Leukocytes generate internal forces(as myosin contracts) to change their shapes and migrate (eg. transendothelial migration of leukocytes ${ }^{[34]}$ ) (Fig. 1). These cells sense the stiffness of the substrate as they continue to migrate $^{[35]}$. As blood cells are born and differentiate in bone marrow (BM), the mechanical environment of $\mathrm{BM}$ influence blood cell fate ${ }^{[36]}$. BM is a highly dynamic tissue composed mostly of hematopoietic cells with a Young's modulus estimated to lie between 15 and 300 $\mathrm{Pa}^{[37,38]}$, which is softer than brain $(0.1 \sim 1.0 \mathrm{kPa})$, skeletal muscle $(8 \sim 17 \mathrm{kPa})$, and bone $(25 \sim 40 \mathrm{kPa})^{[39]}$. Such softness provides a niche for hematopoietic cells ${ }^{[38]}$. In pathophysiology, chronic vascular inflammation is commonly associated with abnormal subendothelial matrix stiffness. However, how matrix stiffness controls leukocyte-substrate adhesion, a critical early step in vascular inflammation, remains poorly understood.

Internal mechanical forces are generated by nonmuscle myosin- II (NMM- II), which is essential for blood cell differentiation. NMM- II is also required for exocytosis, immunological synapse stabilization, and motility of leukocytes ${ }^{[38]}$. A single myosin molecule can generate 1 5 pN force, and multiple myosin molecule can sufficiently induce conformational changes of a protein molecule, which elicits a biochemical signal ${ }^{[40]}$.
In summary, blood cells are affected by timeand load-dependent external and internal mechanical forces and combinations of such forces with other factors such as cytokines, which eventually determines blood cells fate, being either normal or pathophysiological.

\section{Blood megakaryocytes and platelets}

Megakaryocytes (MKs) originate in the hematopoietic stem cell in BM and are then exposed to shear flow $\left(1.3 \sim 4.1 \mathrm{dyn} / \mathrm{cm}^{2}\right)$ as they migrate into circulation to release pro- and pre-platelets into circulating blood $^{[41,42]}$ (Fig. 1). Shear forces promote DNA synthesis, polyploidization, and maturation in MKs, and platelet biogenesis. MK exposure to shear forces leads to phosphorylation of JNK, AP-1 (JunD and c-Fos) up-regulation, and downstream transcriptional changes (ATF4, JUNB, JUN, FOSB, FOS, and JUND) that promote MKs maturation and platelet release. JNK inhibition reduces shear-induced JunD expression ${ }^{[43]}$. However, the molecular mechanisms of shear forces transmission to signal events are not known. Nevertheless scientists utilize these unique properties to produce functional platelets for transfusion from cultured MKs using microfluidic bioreactor, however, the efficiency of this method is currently still $\operatorname{low}^{[44,45]}$. The lung is also a niche for thrombopoiesis. Interestingly, mutations of myosin heavy chain 9 in MKs are associated with May-Hegglin anomaly, which is characterized by low platelet count (thrombocytopenia) and giant platelets ${ }^{[46]}$. These observations suggest a role of mechanotransduction in platelet biogenesis ${ }^{[47]}$.

Blood flow also influences platelet activation, thrombus growth, and stabilization ${ }^{[48,49]}$. In shear flow, the force on a von Willebrand factor (vWF) multimer alters the extent of vWF self-association and induces unfolding of A2 domain to expose cleavage site for ADAMTS $13^{[50,51]}$. Integrin receptors are also mechanosensitive $^{[52,53]}$.

At a low to intermediate shear $(<1000 / \mathrm{s})$ (typically found in veins and larger arteries) the platelet aggregation is predominantly mediated by integrin ${ }^{\alpha} \mathrm{IIb}^{\beta} 3$. At high shear(1000-10,000/s) typically found in the arterial microcirculation or in regions of moderate arterial stenosis, platelet-platelet interactions become progressively more VWF-dependent. At extremely high shear $(10,000-40,000 / \mathrm{s})$ found in severe vessel narrowing by atherothrombosis, platelet aggregation is exclusively mediated by vWF-GPIb $\alpha$ adhesive bonds. Therefore, understanding of mechanotransduction mechanism in thromboembolic events under shear forces is important for future development of therapeutics $^{[54]}$. 


\section{ERYTHROCYTES}

The mechanical features of RBC are of physiological and pathological significance (Fig. 2). RBCs are easily deformed by fluid flow and can pass through the microcirculation due to their large deformability ${ }^{[55]}$. RBCs are more deformable from 37.0 to $42.0^{\circ} \mathrm{C}^{[56]}$. When deformed, RBCs release various molecules and regulate their concentrations in blood. RBCs can be stiffened from malaria by Plasmodium falciparum infection leading to microvascular occlusion and subsequent organ damage ${ }^{[57]}$. Storaged RBCs decrease the deformability in a time-dependent manner, thereby inhibiting microvascular flow, reducing posttransfusion survival, and causing complications. Therefore, the mechanical changes of blood-banked RBCs can limit transfusion ability ${ }^{[3]}$, although concentrations of various molecules change during storage to ${ }^{[58]}$. The deformation induces ATP release (mechanism unknown), which regulates microvascular tone and plays essential roles in vascular physiopathology ${ }^{[55]}$. Extremely high tension may cause haemolysis ${ }^{[59]}$. Osmotic pressure finely tunes the volume of RBCs (in diseases such as jaundice or in different nutritional situations) and changes behaviour of RBCs. In extreme cases, osmotic shock can trigger rupture of red cells and cause lysis ${ }^{[60]}$.

Since mechanically activated Piezo cation channels are expressed on the RBC plasma membrane,

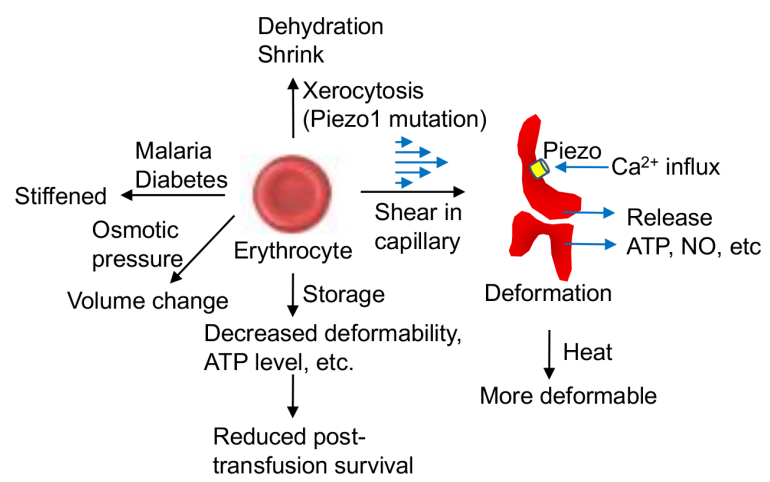

Fig. 2. The mechanical features of a red blood cell (RBC) are of physiological and pathological significance. RBCs are easily deformed by fluid flow and can pass through the capillary due to their large deformability. During deformation, and RBC releases various molecules including ATP and NO. Mechanically activated Piezo cation channels regulates $\mathrm{Ca}^{2+}$ influx (right). Malaria infection and diabetes induce stiffening of RBC and reduce deformability (left). Change of osmosis due to disease or nutritional situations leads to volume changes of RBC (lower left). Storage of RBC also decreases its deformability in time-dependent manner, thereby inhibiting microvascular flow, reducing posttransfusion survival, and causing complications (bottom). Muation of Piezol channel is associated with the dehydrating $\mathrm{RBC}$ disease xerocytosis, implicating a role of mechanotransduction in $\mathrm{RBC}$ volume regulation (top). it is believed that calcium influx into the cells is mechanosensitive ${ }^{[6]]}$. Gain-of-function mutations in Piezol lead to dehydrating RBC disease xerocytosis by increasing robust calcium entry in response to mechanical stretch ${ }^{[62]}$. Conversely, Piezol conditional knockout mice exhibit increased RBCs size and osmotic fragility ${ }^{[63]}$. These results imply a role of mechanotransduction in regulation of RBCs volume homeostasis.

\section{Eosinophil}

Eosinophil is responsible for combating infection and for inflammatory responses such as asthma and allergy. In vitro experiments demonstrate that a shortterm (15 min) cyclic stretch inhibits leukotriene C4 synthesis in eosinophils, when either calcium ionophore A-23187 or chemotactic peptide N-FormylMet- Leu-Phe was simultaneously stimulated, whereas stretching alone showed no effects ${ }^{[64]}$. Adhesion of eosinophils to endothelial cells through $\mathrm{E}$-selectin in the presence of shear enhances transmigration of eosinophil with increased phosphorylation of extracellular signal-regulated kinase (ERK) 2 in cytokine-stimulated endothelial cells. Disassembly of actin cytoskeleton with latrunculin A prevents ERK2 phosphorylation after adhesion under flow conditions, implying a role for the cytoskeleton in mechanosensing in endothelial cells, which play a role in regulating subsequent eosinophil transmigration ${ }^{[65]}$.

\section{Basophil}

Not a study has been done to investigate mechanical effects on function of basophil to date.

\section{Lymphocytes}

Circulating $\mathrm{T}$ lymphocytes attach on the surface of blood vessels during flow and move upstream against the direction of flow (mechanotaxis) ${ }^{[66]}$. Interestingly, this upstream migration is not regulated by classical guidance signaling pathways, and changes in the direction or intensity of flow do not trigger calcium signaling. Instead, upstream flow mechanotaxis of lymphocytes may only rely on a passive self-steering mechanism using the cell rear uropods as wind vanes ${ }^{[6]}$. The following lymphocyte transmigration is also promoted by shearinduced mechanical signals ${ }^{[67]}$. T lymphocytes are activated when the $\mathrm{T}$ cell receptor (TCR) recognizes peptides bound to major histocompatibility complexes on an antigen-presenting cell (APC). Shear forces may regulate enrichment of TCR to lipid rafts and facilitate binding to $\mathrm{APC}^{[68]}$ (Fig. 3). In addition, TCR itself has been shown to be an anisotropic mechanosensor ${ }^{[69]}$. Insideout signal also activates TCR by forces generated by 

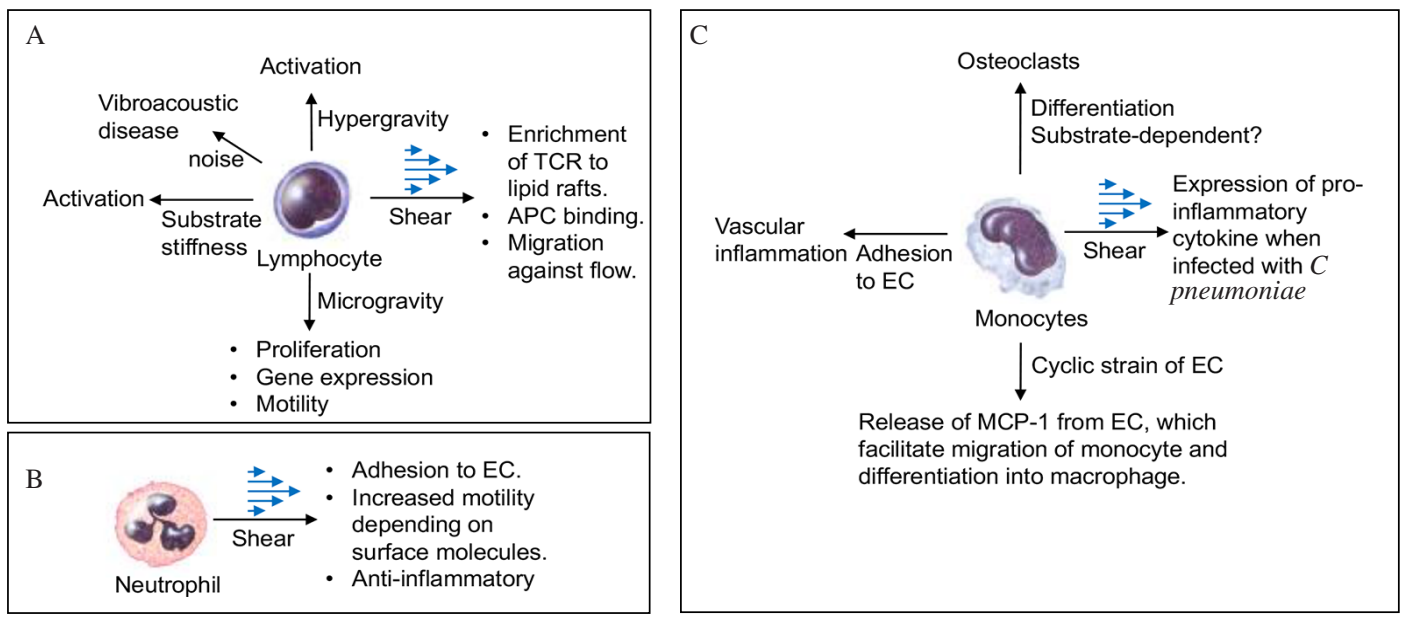

Fig 3. Mechanostransduction in leukocytes. (A) Lymphocytes respond to changes of gravity, low-frequency noise, and shear flow. Substrate stiffness also controls T-cell activation. (B) Shear force regulates adhesion of neutrophils to endothelial cell (EC) and migration. Normal shear reduces pseudopod activity, surface expression of CD18 integrin, and F-actin content, which is an anti-inflammatory measure. (C) Mechanical stimulation releases monocyte chemoattractant protein $1(\mathrm{MCP}-1)$, a potent proinflammatory chemokine from vascular cells. $\mathrm{MCP}-1$ facilitates the migration of monocytes into the subintimal space, where they differentiate into macrophages (bottom). Shear force directly activate monocytes when they are infected with C. pneumoniae (right). Monocytes also adhere EC and senses stiffness of substrate to induce inflammation and differentiation (left and top).

the actomyosin cytoskeleton. The cytoskeletal forces are sufficient for receptor activation in T-cells, which are sensitive to the stiffness of the substrate ${ }^{[70,71]}$. The murine hypertrophic scar model demonstrates that mechanical forces prolong acute inflammation through T-cell-dependent pathways during scar formation $^{[72]}$. These results suggest that targeting these mechanotransduction pathways may be able to alleviate fibrosis.

Gravity is another force that affects lymphocyte activation and spaceflight alters gene expression during $\mathrm{T}$-cell activation ${ }^{[73-78]}$. Exposure to microgravity created by rotating wall vessels reduces lymphocyte migration $^{[79]}$. However, direct observation of cells in suspension in true microgravity shows that lymphocytes are more motile than on the ground (Cogoli-Greuter et al. 1996). Similar to microgravity, hypergravity has been found to influence $T$ cell activation and proliferation, and speed up development of the motility ${ }^{[30]}$.

\section{Neutrophil}

Neutrophils are the most abundant of circulating leukocytes, and the human body replaces about one million neutrophils per second. Once activated, neutrophils project pseudopods and bind to other blood cells $^{[11]}$. Therefore, neutrophil activation significantly affects microvascular flow. In general shear, stress is anti-inflammatory for neutrophils and reduces their pseudopod activity, F-actin content, and surface expression of integrins ${ }^{[80]}$. The shear-driven oscillation of neutrophils between the activated and resting states is dependent on the presence of other factors such as platelet activating factor $(\mathrm{PAF})^{[81]}$.
On stiff substrate (12 kPa), integrin engagement is essential for motility, but not on soft hydrogels $(2 \mathrm{kPa})$. During three-dimensional movement of neutrophils, integrins are not necessary for migration ${ }^{[82]}$. These results suggest that neutrophil chemotaxis is markedly different depending on the mechanical environment ${ }^{[83]}$. Recently it was demonstrated that GEF-H1 is required for shear stress-induced migration during inflammation and shear force induces relocalization of GEF-Hl to uropods ${ }^{[84]}$.

\section{Monocytes}

Mechanical forces (eg. cyclic streaching) stimulate vascular cells to release monocyte chemoattractant protein $1(\mathrm{MCP}-1)$ (a potent proinflammatory chemokine). Hypertension up-regulates expression of MCP-1, but reducing blood pressure through pharmacological intervention normalizes the response, implying mechanoregulation of $\mathrm{MCP}-1$ expression. MCP-1 facilitates the migration of monocytes into the subintimal space, where they differentiate into macrophages $^{[85]}$. Macrophages are also mechanosensitive ${ }^{[86]}$. On the other hand, monocyte proliferation and differentiation to osteoclasts is successfully achieved by culturing on poly-dimethyl siloxane culture surface coated with collagen ${ }^{[87]}$. However, how substrate stiffness regulates the differentiation is not known. Physiological level of shear stress stimulates expression of pro-inflammatory cytokine in C. pneumoniaeinfected, but not in uninfected, human monocytes ${ }^{[88]}$. In addition, substrate stiffness regulates monocyteendothelial adhesion via Rho-mediated ICAM-1 clustering in biphasic fashion: namely both matrix 
softening and stiffening increase adhesive interac$\operatorname{tion}^{[35]}$. These results suggest that mechanotransduction is an important determinant of inflammatory response to infection and vascular inflammation.

\section{Molecular mechanism of how forces convert to biochemical signaling}

Elucidating the molecular mechanism of mechanotransduction is essential for a clear understanding of mechano-biology and eventually for developing pharmacological interventions. However, very little is known about:1) Which molecules are involved in the pathways;2) How signals are received and transmitted and 3) The structural basis behind this biological phenomenon as a function of time. Scientists hypothesize that mechanical forces are received by transmembrane proteins such as integrins, glycocalicin, and channels, and forces are transmitted through the cytoskeleton at sites of connection to focal adhesions, nucleus, and neighboring cells ${ }^{[89]}$. During the transmission and conversion to biochemical signals, conformational changes of molecules are believed to occur. However, one major obstacle is that it is difficult to maintain a stressed state for a molecule in vitro. In order to overcome the burden, scientists use both top-down (forward genetics) and bottomup (reverse genetics) approaches. The top-down approach tries to identify a mechanosensing molecule using knockdown or knockout strategies (eg. piezo channel) ${ }^{[00]}$. The bottom-up approach speculates mechanosensing function based on atomic structure of a molecule and computational analysis (eg. von Willebrand factor, talin, and filamin) ${ }^{[40,50,91,92]}$ (Fig. 4). vWF is secreted from vascular endothelial cells into the blood and circulates in plasma as a multimeric molecule. Elevated shear forces induce unfolding of A2 domain, exposing binding site for GPIb $\alpha$ receptor in $\mathrm{Al}$ domain, cryptic ADAMTS13 binding sites, and cleavage site in A2 domain ${ }^{[50,93]}$ (Fig. $4 \boldsymbol{A}$ ). The structure of full-length Piezol channel has recently been revealed using cryo-electron miscrocopy ${ }^{[94]}$. Piezol forms a trimeric propeller-like structure with 14 apparently resolved segments in transmembrane domain per subunit (Fig. 4B). This trimeric architecture suggests that Piezol may use its peripheral regions as force sensors to gate the central pore channel, but this hypothesis has never been tested in a purified lipid bilayer ${ }^{[05]}$. Talin is highly concentrated at focal adhesion (FA) to directly connect actin filaments to FA proteins including vinculin and integrins $^{[91,96]}$. Externally or internally generated-forces are transmitted to talin via actin filaments and expose vinculin binding site (Fig. 4 C). However, it remains to be definitively shown that this indeed occurs in living cells. Filamin A (FLNA) is a homo dimeric actin crosslinking protein comprised of a spectrin-like actinbinding domain and 24 immunoglobulin-like repeats $(\mathrm{R} 1-24)^{[40]}$. Force-induced conformational changes in FLNA "mechanosensing domains" can regulate partner
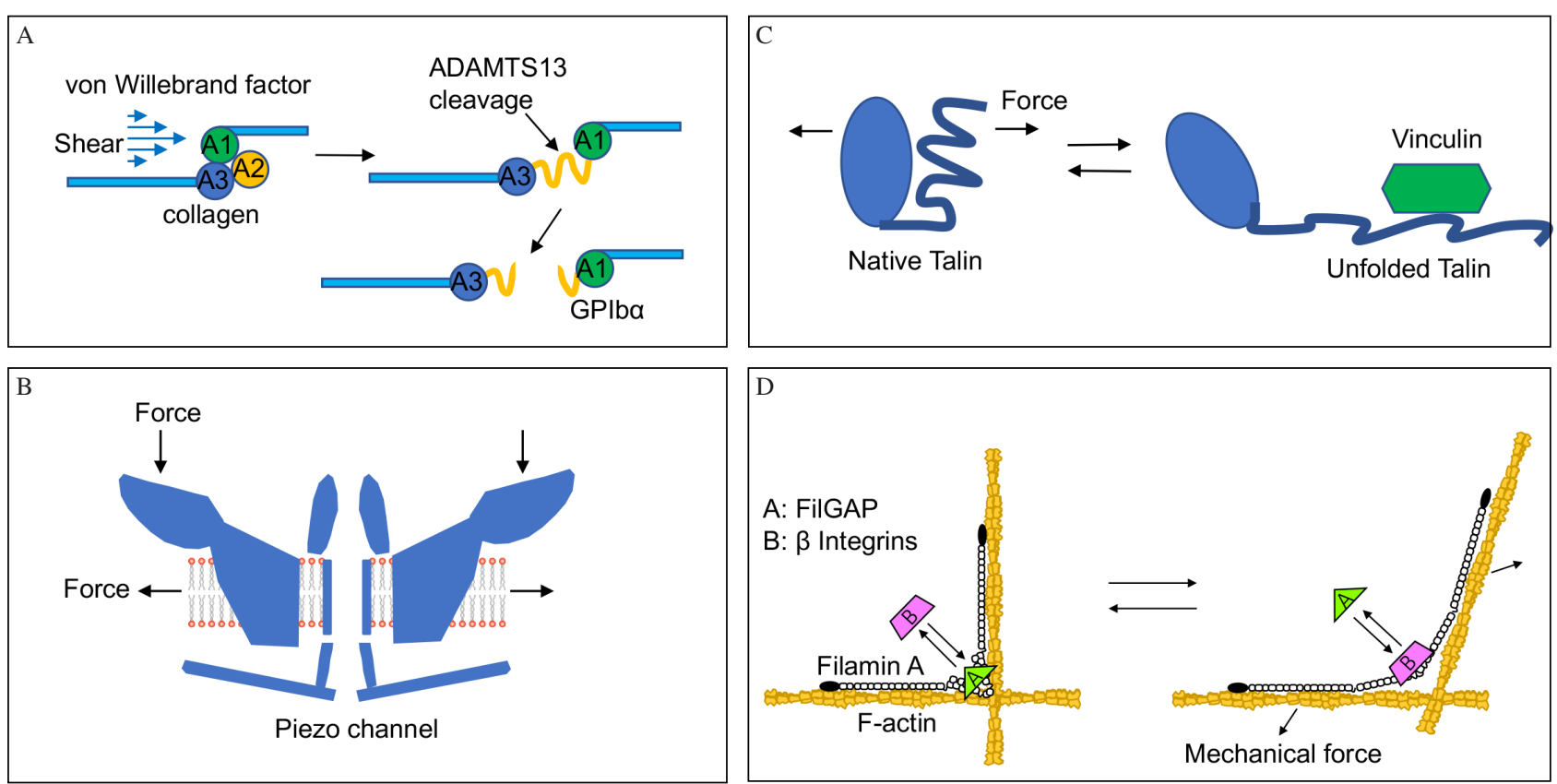

Fig. 4. Schematic models of mechanosensing molecules and how mechanical forces are converted to biochemical signals. (A) Shear forces expose ADAMTS13 cleavage site of Von Willebrand factor (vWF) and allow binding to GPIb $\alpha$ receptor on platelets. (B) A model of the force-induced gating of Piezo channel. (C) Mechanical force induces conformational changes of talin to expose vinculin-binding site. (D) Differential mechanotransduction in filamin A occurs through opening cryptic sites (A: eg. integrin) and spatial separation of binding sites (B: eg. FilGAP). F-actin, filamentous actin. 
interactions by two distinctive mechanisms: 1) exposing a cryptic integrin binding site in R21; and 2) avidity loss caused by separating subunit chains, which move paired R23s apart and dissociate a Rac-GAP (FilGAP) ${ }^{[97]}$ (Fig. 4D). A microscopic method used to monitor R21 exposure documented spatial and temporal R21 exposure in living cells ${ }^{[98]}$.

\section{ACKNOWLEDGEMENTS}

I thank Dr. Monika Pilichowska from Tufts Medical Center, Boston, MA, USA for her critical reading and suggestions.

\section{References}

[1] Murrell M, Oakes PW, Lenz M, et al. Forcing cells into shape: the mechanics of actomyosin contractility. Nat Rev Mol Cell Biol, 2015;16(8):486-98.

[2] Baeyens N, Schwartz MA. Biomechanics of vascular mechanosensation and remodeling. Mol Biol Cell, 2016;27(1):7-11.

[3] Yalcin O, Ortiz D, Tsai AG, et al. Microhemodynamic aberrations created by transfusion of stored blood. Transfusion, 2014;54(4):1015-27.

[4] Matsumoto T, Abe H, Ohashi T, et al. Local elastic modulus of atherosclerotic lesions of rabbit thoracic aortas measured by pipette aspiration method. Physiol Meas, 2002;23(4):635-48.

[5] Georges PC, Hui JJ, Gombos Z, et al. Increased stiffness of the rat liver precedes matrix deposition: implications for fibrosis. Am J Physiol Gastrointest Liver Physiol, 2007;293(6):G1147-54.

[6] Martin P. Wound healing--aiming for perfect skin regeneration. Science, 1997;276(5309):75-81.

[7] Wang L, Luo JY, Li B, et al. Integrin-YAP/TAZ-JNK cascade mediates atheroprotective effect of unidirectional shear flow. Nature, 2016,Dec 7. doi: 10.1038/ nature20602. [Epub ahead of print]

[8] Ciciliano JC, Tran R, Sakurai Y, et al. The platelet and the biophysical microenvironment: lessons from cellular mechanics. Thromb Res, 2014;133(4):532-7.

[9] Engler AJ, Sen S, Sweeney HL, et al. Matrix elasticity directs stem cell lineage specification. Cell, 2006;126(4):677-89.

[10] Martini J, Cabrales P, Tsai AG, et al. Mechanotransduction and the homeostatic significance of maintaining blood viscosity in hypotension, hypertension and haemorrhage. J Intern Med, 2006;259(4):364-72.

[11] Ekpenyong AE, Toepfner N, Chilvers ER, et al. Mechanotransduction in neutrophil activation and deactivation. Biochim Biophys Acta, 2015;1853(11 Pt B):3105-16.

[12] Fu Y, Hou Y, Fu C, et al. A novel mechanism of gamma/ delta T-lymphocyte and endothelial activation by shear stress: the role of ecto-ATP synthase beta chain. Circ Res, 2011;108(4):410-7.

[13] Burns AR, Zheng Z, Soubra SH, et al. Transendothelial flow inhibits neutrophil transmigration through a nitric oxide-dependent mechanism: potential role for cleft shear stress. Am J Physiol Heart Circ Physiol, 2007;293(5):H2904-10.

[14] Seneviratne A, Hulsmans M, Holvoet P, et al. Biomechanical factors and macrophages in plaque stability. Cardiovasc Res, 2013;99(2):284-93.

[15] Liu HB, Zhang J, Xin SY, et al. Mechanosensitive properties in the endothelium and their roles in the regulation of endothelial function. J Cardiovasc Pharmacol, 2013;61(6):461-70.

[16] Hahn C, Schwartz MA. Mechanotransduction in vascular physiology and atherogenesis. Nat Rev Mol Cell Biol, 2009;10(1):53-62.

[17] Abe J, Berk BC. Novel mechanisms of endothelial mechanotransduction. Arterioscler Thromb Vasc Biol, 2014;34(11):2378-86.

[18] Dorland YL, Huveneers S. Cell-cell junctional mechanotransduction in endothelial remodeling. Cell Mol Life Sci, 2017;74(2):279-92.

[19] Klabunde RE. 2012. Cardiovascular Physiology Concepts: Lippincott Williams \& Wilkins

[20] Pendergast DR, Moon RE, Krasney JJ, et al. Human Physiology in an Aquatic Environment. Compr Physiol, 2015;5(4):1705-50.

[21] Bianchi E, Molteni R, Pardi R, et al. Microfluidics for in vitro biomimetic shear stress-dependent leukocyte adhesion assays. J Biomech, 2013;146(2):276-83.

[22] Zhou J, Li YS, Chien S. Shear stress-initiated signaling and its regulation of endothelial function. Arterioscler Thromb Vasc Biol, 2014;34(10):2191-8.

[23] Cheng C, Tempel D, van Haperen R, et al. Atherosclerotic lesion size and vulnerability are determined by patterns of fluid shear stress. Circulation, 2006;113(23):2744-53.

[24] Van der Heiden K, Gijsen FJ, Narracott A, et al. The effects of stenting on shear stress: relevance to endothelial injury and repair. Cardiovasc Res, 2013,99(2):269-75.

[25] Papaioannou TG, Stefanadis C. Vascular wall shear stress: basic principles and methods. Hellenic J Cardiol, 2005;46(1):9-15.

[26] Li X, Yang Q, Wang Z, et al. Shear stress in atherosclerotic plaque determination. DNA Cell Biol, 2014;33(12):830-8.

[27] Korin N, Kanapathipillai M, Matthews BD, et al. Shearactivated nanotherapeutics for drug targeting to obstructed blood vessels. Science, 2012;337(6095):738-42.

[28] Cogoli A, Tschopp A, Fuchs-Bislin P. Cell sensitivity to gravity. Science, 1984;225(4658):228-30.

[29] Pippia P, Sciola L, Cogoli-Greuter M, et al. Activation signals of T lymphocytes in microgravity. J Biotechnol, 1996;47(2-3):215-22.

[30] Galimberti M, Tolic-Norrelykke IM, Favillini R, et al. Hypergravity speeds up the development of T-lymphocyte motility. Eur Biophys J, 2006;35(5):393-400.

[31] Najrana T, Sanchez-Esteban J. Mechanotransduction as an Adaptation to Gravity. Front Pediatr, 2016;4140.

[32] Castro AP, Aguas AP, Grande NR, et al. Increase in 
CD8+ and CD4+ T lymphocytes in patients with vibroacoustic disease. Aviat Space Environ Med, 1999;70(3 Pt 2):A141-4.

[33] Alves-Pereira M, Castelo Branco NA. Vibroacoustic disease: biological effects of infrasound and low-frequency noise explained by mechanotransduction cellular signalling. Prog Biophys Mol Biol, 2007;93(1-3):256-79.

[34] Schaefer A, Hordijk PL. Cell-stiffness-induced mechanosignaling - a key driver of leukocyte transendothelial migration. J Cell Sci, 2015;128(13):2221-30.

[35] Scott HA, Quach B, Yang X, et al. Matrix stiffness exerts biphasic control over monocyte-endothelial adhesion via Rho-mediated ICAM-1 clustering. Integr Biol (Camb), 2016;8(8):869-78.

[36] Gurkan UA, Akkus O. The mechanical environment of bone marrow: a review. Ann Biomed Eng, 2008;36(12):1978-91.

[37] Choi YS, Vincent LG, Lee AR, et al. Mechanical derivation of functional myotubes from adipose-derived stem cells. Biomaterials, 2012;33(8):2482-91.

[38] Shin JW, Swift J, Ivanovska I, et al. Mechanobiology of bone marrow stem cells: from myosin-II forces to compliance of matrix and nucleus in cell forms and fates. Differentiation, 2013;86(3):77-86.

[39] Discher DE, Janmey P, Wang YL. Tissue cells feel and respond to the stiffness of their substrate. Science, 2005;310(5751):1139-43.

[40] Nakamura F, Stossel TP, Hartwig JH. The filamins: organizers of cell structure and function. Cell Adh Migr, 2011;5(2):160-9.

[41] Junt T, Schulze H, Chen Z, et al. Dynamic visualization of thrombopoiesis within bone marrow. Science, 2007;317(5845):1767-70.

[42] Dunois-Larde C, Capron C, Fichelson S, et al. Exposure of human megakaryocytes to high shear rates accelerates platelet production. Blood, 2009;114(9):1875-83.

[43] Luff SA, Papoutsakis ET. Megakaryocytic maturation in response to shear flow is mediated by the activator protein $1(\mathrm{AP}-1)$ transcription factor via mitogen-activated protein kinase (MAPK) mechanotransduction. J Biol Chem, 2016;291(15):7831-43.

[44] Thon JN, Medvetz DA, Karlsson SM, et al. Road blocks in making platelets for transfusion. J Thromb Haemost, 2015;13 Suppl 1S55-62.

[45] Wang B, Zheng J. Platelet generation in vivo and in vitro. Springerplus, 2016;5(1):787.

[46] Kelley MJ, Jawien W, Ortel TL, et al. Mutation of MYH9, encoding non-muscle myosin heavy chain A, in May-Hegglin anomaly. Nat Genet, 2000;26(1):106-8.

[47] Weyrich AS, Zimmerman GA. Platelets in lung biology. Annu Rev Physiol, 2013;75569-91.

[48] Cosemans JM, Angelillo-Scherrer A, Mattheij NJ, et al. The effects of arterial flow on platelet activation, thrombus growth, and stabilization. Cardiovasc Res, 2013;99(2):342-52.

[49] Qiu Y, Ciciliano J, Myers DR, et al. Platelets and physics: How platelets "feel" and respond to their mechanical microenvironment. Blood Rev, 2015;29(6):377-86.

[50] Zhang X, Halvorsen K, Zhang CZ, et al. Mechanoenzymatic cleavage of the ultralarge vascular protein von Willebrand factor. Science, 2009;324(5932):1330-4.

[51] Dayananda KM, Singh I, Mondal N, et al. von Willebrand factor self-association on platelet GpIbalpha under hydrodynamic shear: effect on shear-induced platelet activation. Blood, 2010;116(19):3990-8.

[52] Goncalves I, Nesbitt WS, Yuan Y, et al. Importance of temporal flow gradients and integrin alphaIIbbeta3 mechanotransduction for shear activation of platelets. J Biol Chem, 2005;280(15):15430-7.

[53] Dong X, Zhao B, Iacob RE, et al. Force interacts with macromolecular structure in activation of TGF-beta. Nature, 2017;542(7639):55-9.

[54] Jackson SP, Nesbitt WS, Westein E. Dynamics of platelet thrombus formation. J Thromb Haemost, 2009; 7 (Suppl): 117-20.

[55] Wan J, Forsyth AM, Stone HA. Red blood cell dynamics: from cell deformation to ATP release. Integr Biol (Camb), 2011;3(10):972-81.

[56] Foo JJ, Chan V, Feng ZQ, et al. Human red blood cells deformed under thermal fluid flow. Biomed Mater, 2006;1(1):1-7.

[57] van der Heyde HC, Nolan J, Combes V, et al. A unified hypothesis for the genesis of cerebral malaria: sequestration, inflammation and hemostasis leading to microcirculatory dysfunction. Trends Parasitol, 2006;22(11):503-8.

[58] Serrano K, Levin E, Chen D, et al. An investigation of red blood cell concentrate quality during storage in paediatric-sized polyvinylchloride bags plasticized with alternatives to di-2-ethylhexyl phthalate (DEHP). Vox Sang, 2016;110(3):227-35.

[59] Leverett LB, Hellums JD, Alfrey CP, et al. Red blood cell damage by shear stress. Biophys $J$, 1972;12(3):257-73.

[60] Lang F, Lang KS, Lang PA, et al. Osmotic shockinduced suicidal death of erythrocytes. Acta Physiol (Oxf), 2006;187(1-2):191-8.

[61] Brain MC, Pihl C, Robertson L, et al. Evidence for a mechanosensitive calcium influx into red cells. Blood Cells Mol Dis, 2004;32(3):349-52.

[62] Cahalan SM, Lukacs V, Ranade SS, et al. Piezol links mechanical forces to red blood cell volume. Elife, $2015 ; 4$

[63] Lukacs V, Mathur J, Mao R, et al. Impaired PIEZO1 function in patients with a novel autosomal recessive congenital lymphatic dysplasia. Nat Commun, 2015;68329.

[64] Waters CM, Sporn PH, Liu M, et al. Cellular biomechanics in the lung. Am J Physiol Lung Cell Mol Physiol, 2002;283(3):L503-9.

[65] Cuvelier SL, Paul S, Shariat N, et al. Eosinophil adhesion under flow conditions activates mechanosensitive signaling pathways in human endothelial cells. $J$ Exp Med, 2005;202(6):865-76. 
[66] Valignat MP, Negre P, Cadra S, et al. Lymphocytes can self-steer passively with wind vane uropods. Nat Commun, 2014;55213.

[67] Cinamon G, Shinder V, Alon R. Shear forces promote lymphocyte migration across vascular endothelium bearing apical chemokines. Nat Immunol, 2001;2(6):515-22.

[68] Lanzavecchia A, Iezzi G, Viola A. From TCR engagement to $\mathrm{T}$ cell activation: a kinetic view of $\mathrm{T}$ cell behavior. Cell, 1999;96(1):1-4.

[69] Kim ST, Takeuchi K, Sun ZY, et al. The alphabeta T cell receptor is an anisotropic mechanosensor. J Biol Chem, 2009;284(45):31028-37.

[70] Hui KL, Balagopalan L, Samelson LE, et al. Cytoskeletal forces during signaling activation in Jurkat T-cells. Mol Biol Cell, 2015;26(4):685-95.

[71] Judokusumo E, Tabdanov E, Kumari S, et al. Mechanosensing in T lymphocyte activation. Biophys $J$, 2012;102(2):L5-7.

[72] Wong VW, Paterno J, Sorkin M, et al. Mechanical force prolongs acute inflammation via T-celldependent pathways during scar formation. FASEB J, 2011;25(12):4498-510.

[73] Cogoli A, Cogoli-Greuter M. Activation and proliferation of lymphocytes and other mammalian cells in microgravity. Adv Space Biol Med, 1997;633-79.

[74] Benavides Damm T, Walther I, Wuest SL, et al. Cell cultivation under different gravitational loads using a novel random positioning incubator. Biotechnol Bioeng, 2014;111(6):1180-90.

[75] Hauschilda S, Taubera S, Laubera B, et al. T cell regulation in microgravity-The current knowledge from in vitro experiments conducted in space, parabolic flights and ground-based facilities. Acta Astronautica, 2014;104(1):365-77.

[76] Martinez EM, Yoshida MC, Candelario TL, et al. Spaceflight and simulated microgravity cause a significant reduction of key gene expression in early T-cell activation. Am J Physiol Regul Integr Comp Physiol, 2015;308(6):R480-8.

[77] Hughes-Fulford M, Chang TT, Martinez EM, et al. Spaceflight alters expression of microRNA during T-cell activation. FASEB J, 2015;29(12):4893-900.

[78] Chen Y, Xu C, Wang P, et al. Effect of Long-Term Simulated Microgravity on Immune System and Lung Tissues in Rhesus Macaque. Inflammation, 2017,

[79] Plett PA, Abonour R, Frankovitz SM, et al. Impact of modeled microgravity on migration, differentiation, and cell cycle control of primitive human hematopoietic progenitor cells. Exp Hematol, 2004;32(8):773-81.

[80] Zhang X, Cheng R, Rowe D, et al. Shear-sensitive regulation of neutrophil flow behavior and its potential impact on microvascular blood flow dysregulation in hypercholesterolemia. Arterioscler Thromb Vasc Biol, 2014;34(3):587-93.

[81] Mitchell MJ, Lin KS, King MR. Fluid shear stress increases neutrophil activation via platelet-activating factor. Biophys J, 2014;106(10):2243-53.

[82] Lammermann T, Bader BL, Monkley SJ, et al. Rapid leukocyte migration by integrin-independent flowing and squeezing. Nature, 2008;453(7191):51-5.

[83] Jannat RA, Robbins GP, Ricart BG, et al. Neutrophil adhesion and chemotaxis depend on substrate mechanics. $J$ Phys Condens Matter, 2010;22(19):194117.

[84] Fine N, Dimitriou ID, Rullo J, et al. GEF-H1 is necessary for neutrophil shear stress-induced migration during inflammation. J Cell Biol, 2016;215(1):107-19.

[85] Guest TM, Vlastos G, Alameddine FM, et al. Mechanoregulation of monocyte chemoattractant protein-1 expression in rat vascular smooth muscle cells. Antioxid Redox Signal, 2006;8(9-10):1461-71.

[86] Adlerz KM, Aranda-Espinoza H, Hayenga HN. Substrate elasticity regulates the behavior of human monocytederived macrophages. Eur Biophys J, 2016;45(4):301-9.

[87] Shafieyan Y, Tiedemann K, Goulet A, et al. Monocyte proliferation and differentiation to osteoclasts is affected by density of collagen covalently bound to a poly(dimethyl siloxane) culture surface. J Biomed Mater Res A, 2012;100(6):1573-81.

[88] Cheeniyil A, Evani SJ, Dallo SF, et al. Shear stress upregulates IL-1beta secretion by Chlamydia pneumoniae- infected monocytes. Biotechnol Bioeng, 2015;112(4):838-42.

[89] Salameh A, Dhein S. Effects of mechanical forces and stretch on intercellular gap junction coupling. Biochim Biophys Acta, 2013;1828(1):147-56.

[90] Coste B, Mathur J, Schmidt M, et al. Piezol and Piezo2 are essential components of distinct mechanically activated cation channels. Science, 2010;330(6000):55-60.

[91] Haining AW, Lieberthal TJ, Del Rio Hernandez A. Talin: a mechanosensitive molecule in health and disease. FASEB J, 2016;30(6):2073-85.

[92] Razinia Z, Makela T, Ylanne J, et al. Filamins in mechanosensing and signaling. Annu Rev Biophys, 2012;41227-46.

[93] Crawley JT, de Groot R, Xiang Y, et al. Unraveling the scissile bond: how ADAMTS13 recognizes and cleaves von Willebrand factor. Blood, 2011;118(12):3212-21.

[94] Ge J, Li W, Zhao Q, et al. Architecture of the mammalian mechanosensitive Piezol channel. Nature, 2015;527(7576):64-9.

[95] Wu J, Lewis AH, Grandl J. Touch, Tension, and Transduction-The Function and Regulation of Piezo Ion Channels. Trends Biochem Sci, 2017;42(1):57-71.

[96] Elosegui-Artola A, Oria R, Chen Y, et al. Mechanical regulation of a molecular clutch defines force transmission and transduction in response to matrix rigidity. Nat Cell Biol, 2016;18(5):540-8.

[97] Ehrlicher AJ, Nakamura F, Hartwig JH, et al. Mechanical strain in actin networks regulates FilGAP and integrin binding to filamin A. Nature, 2011;478(7368):260-3.

[98] Nakamura F, Song M, Hartwig JH, et al. Documentation and localization of force-mediated filamin A domain perturbations in moving cells. Nat Commun, 2014;5:4656.

(Received 13 Juanary 2017, Revised 06 February 2017, Accepted 14 February 2017) 